\title{
Correlation of CT Cerebral Vascular Territories with Function: 3. Middle Cerebral Artery
}

\author{
Stephen A. Berman ${ }^{1}$ \\ L. Anne Hayman ${ }^{2}$ \\ Vincent C. Hinck ${ }^{1}$
}

Schematic displays are presented of the cerebral territories supplied by branches of the middle cerebral artery as they would appear on axial and coronal computed tomographic (CT) scan sections. Companion diagrams of regional cortical function and a discussion of the fiber tracts are provided to simplify correlation of clinical deficits with coronal and axial CT abnormalities.
This report is the third in a series designed to correlate cerebral vascular territories and functional anatomy in a form directly applicable to computed tomography $(\mathrm{CT})$. The illustrations are intended to simplify analysis of CT images in terms of clinical signs and symptoms and vascular territories in everyday practice. The anterior and posterior cerebral arteries have been described [1, 2]. This report deals with the middle cerebral arterial territory.

Knowledge of cerebral vascular territories can help in differentiating between infarction and other pathologic processes. For example, if the position and extent of a lesion and the usual position and extent of a vascular territory are incongruous, infarction should receive relatively low diagnostic priority and vice versa. Knowledge of vascular territories can also facilitate correct interpretation of cerebral angiograms by pinpointing specific vessels for particularly close attention.

Knowledge of functional neuroanatomy applied to a patient's clinical findings can improve detection of subtle lesions by pinpointing specific areas for special attention on CT and specific vessels for attention on angiograms.

\section{Discussion}

The largest area of the brain that is normally supplied by the vessel(s) of the middle cerebral territory is indicated in figures 1 and 2. An occlusion of the middle cerebral artery will rarely produce a CT lesion larger than that defined in these illustrations. In fact, collateral supply, which often interfaces at the periphery of an infarction, may cause the area of involvement to be smaller than that schematically defined. Furthermore, the area of involvement may have a patchy appearance because only selected branches within the territory are occluded by emboli or fall victim to failure of collateral circulation. Very rarely occlusion may result in infarction of an area that is larger than the territory ascribed to it in figures 1-3. In these cases the territory supplied by the infarcted vessel has "extended" via collateral vessels into adjacent territory(s), the natural supply of which has previously been compromised. Thus when the rescuing vessel is compromised, territory outside the usual vascular limit is also compromised.

The vascular territory generally supplied by the middle cerebral artery and the neurologic functions ascribed thereto are mapped on schematic axial and coronal 
CT scans* (figs. 1 and 2) and on maps of the lateral surface of the left cerebral hemisphere (fig. 3). The branches of the middle cerebral artery have been divided into two groups: (1) the lateral lenticulostriate branches to the basal ganglia and adjacent fiber tracts and (2) the hemispheric branches to the cerebral convexity. Each group is discussed separately.

\section{Lateral Lenticulostriate Arteries}

In $20 \%$ of cases the lateral lenticulostriate branches arise from one of the hemispheric branches of the middle cerebral artery. In the other cases they arise from the trunk of the middle cerebral artery. They supply the substantia innominata, the lateral part of the anterior commissure, most of the putamen and lateral segment of the globus pallidus, the superior half of the internal capsule and adjacent corona radiata, and the body and head (except the anterior inferior portion) of the caudate nucleus. Portions of the optic radiation (which originate from the lateral geniculate body) and the arcuate fasciculus are supplied by the striate branches [4].

The largest area of the brain normally supplied by the striate vessels is shown in figures 1 and 2 . It will be smaller if a large recurrent artery of Heubner, anterior choroidal artery, or both are present, since these vessels may nourish the anterior inferomedial part of the striate territory shown in figures 1 and 2.

The striate branches of the middle cerebral artery are "end branches" in that they have few if any major anastomotic connections other than those of the capillary bed. They do not supply the claustrum and extreme capsule, which are nourished by the hemispheric branches that supply the insular cortex [5] (figs. 4 and 5).

Vascular lesions in the striate distribution can disturb four functions: (1) movement, (2) tactile sensation, (3) speech, and (4) vision. The deficiency of movement is that of contralateral hemiparesis of the face, arm, and leg, $\dagger$ which is caused by damage to the motor fibers in the internal capsule. Weakness, slowness, and changes in passive tone accompany the hemiparesis because the basal ganglia and extrapyramidal pathways are affected. A true parkinsonian syndrome is not a recognized feature of basal ganglia infarction. However, the slowness, increased tone, and spasticity associated with capsular infarction may sometimes be mistaken for early parkinsonism. Indeed, the basal ganglia component of the hemiplegia seen in striate lesions may partly account for the greater degree of spasticity sometimes seen when these lesions are contrasted with hemispheric lesions that produce hemiplegia [6].

The sensory tracts lie close to the motor tracts in the region of the internal capsule. Hence both functions are commonly affected in lesions of the striate territory. Pure motor strokes (involving face, arm, and leg) sometimes occur with striate lesions $[7,8]$. However, this is not diagnostic of a lesion in the striate area. Pure motor stroke may be seen with infarctions at: (1) the base of the pons (due to occlusion of the paramedian branches of the basilar artery), (2) the medullary pyramids (vertebral branches), or (3) rarely at the cerebral peduncle (basilar or posterior cerebral artery) [7-12]. Since the striate branches of the middle cerebral artery may not supply the entire posterior limb of the internal capsule, occlusion of the anterior choroidal artery may be the cause of identical deficits $[7,9,12]$. Occlusion of any of these vessels can cause a very small infarction that may be invisible on CT scans.

Striate lesions that damage the sensory fibers in the posterior limb of the internal capsule cause an increased threshold for the primary modalities of touch, pain, temperature, and vibration in the contralateral face, arm, and leg. Lesions in the posterior cerebral territory in subcortical or thalamic nuclei may mimic these findings [2]. Finally, of course, infarctions of the other vessels that supply the posterior internal capsule can mimic striate artery lesions as previously discussed.

Speech may occasionally be impaired by isolated lesions of the lateral striate territory of the dominant hemisphere [1]. In some instances there is reduced spontaneous verbal output and impaired ability to repeat sentences or name objects, but speech comprehension remains intact. This type of deficit is called Broca aphasia. It occurs frequently with lesions in the dominant cerebral hemisphere and is rarely seen with lesions of the striate territory [13].

Striate lesions may also involve the arcuate fasciculus. This subcortical fiber tract connects the area of the brain labeled "understanding" in figure 3B (the area of Wernicke) with the area labeled "speaking" (the area of Broca). Damage to this tract causes conduction aphasia (an impaired ability to repeat words or phrases) $[14,15]$.

The visual deficit produced by lesions in the striate territory is that of a contralateral homonymous hemianopsia that may be complete or subtle and difficult to detect clinically $[16,17]$. This deficit is caused when there is damage to the optic radiations that traverse the striate territory as they originate from the geniculate body.

\section{Hemispheric Branches}

Twelve major cortical branches are illustrated in figure $3 \mathrm{~A}$. Considering the large number of branches, one might expect a great variety of clinical sequelae. The overall picture can be simplified by comparing the vascular territories of figure $3 \mathrm{~A}$ with the functional areas of figure 3B. Before discussing each functional and vascular territory there are two issues that must be clarified [1]. The areas marked "speaking" and "understanding" are present only in the dominant hemisphere, which is the left hemisphere in almost all right-handed patients and in about one-half of left-handed patients. In the nondominant hemisphere these areas have no well accepted clinically demonstrable function. The cortical area marked "hearing" receives impulses from both ears. Therefore deficits in this area must be bilateral to cause clinical symptoms [2]. The insular cortex is not shown in figure 3 since it lies buried

\footnotetext{
* The plane of coronal CT scans depends on head positioning. If the coronal image is a reconstruction, the plane is perpendicular to the axial scans, which may vary in their orientation. Therefore, we have chosen to illustrate the vascular territories in the anatomic plane. The reader must correct for minor differences in scanning angle. This will be facilitated by a review of coronal slices illustrated by Matsui and Hirano [3].

$\dagger$ "Arm" and "leg" refer to the upper and lower extremities, respectively. They were chosen to simplify the labeling of figure 3 and will be used throughout the text for consistency.
} 

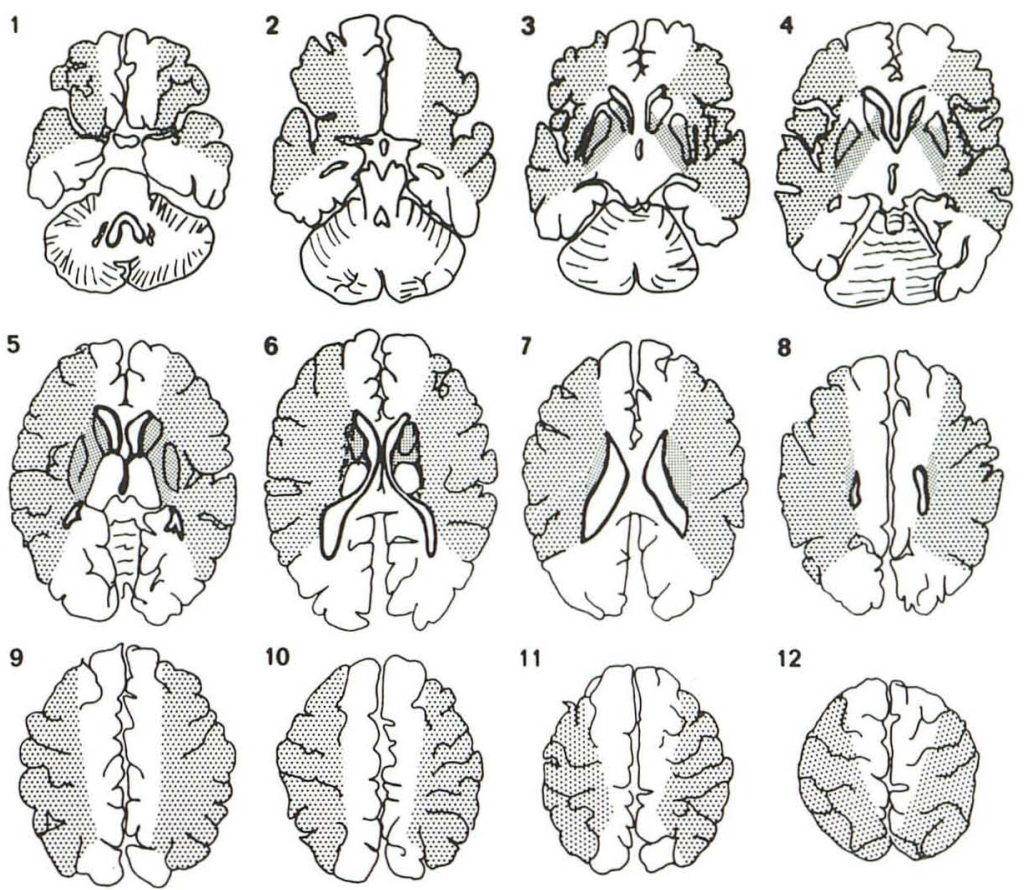

12

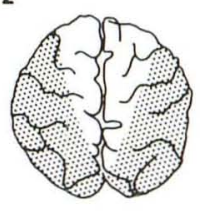

Fig. 1.-Axial CT scan diagrams arranged in sequence from base to vertex. Angle and levels of scan planes are shown in figure 3. Territory of middle cerebral artery is divided into two regions: lateral lenticulostriate (fine stipple) and hemispheric (course stipple).

A

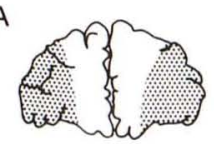

$\mathrm{E}$
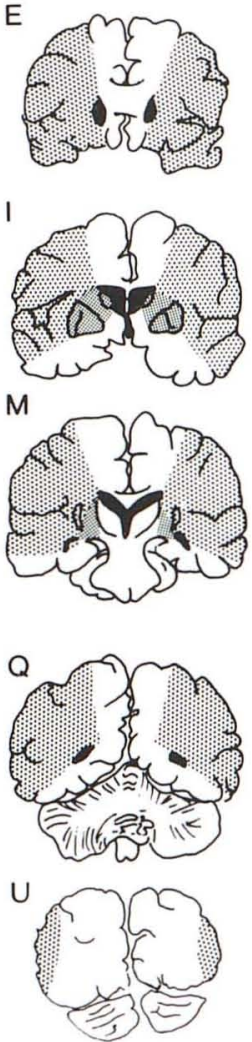

B

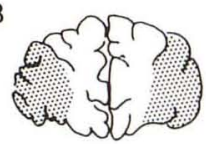

F
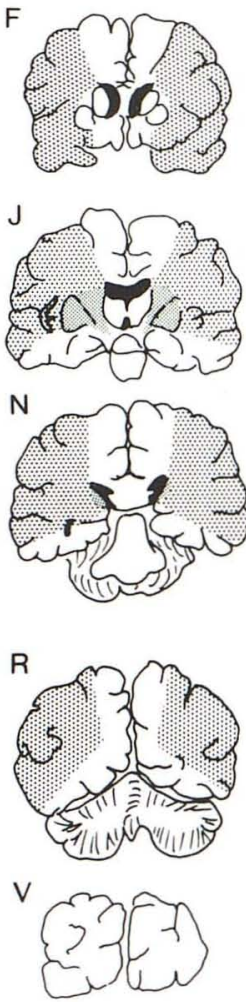

C
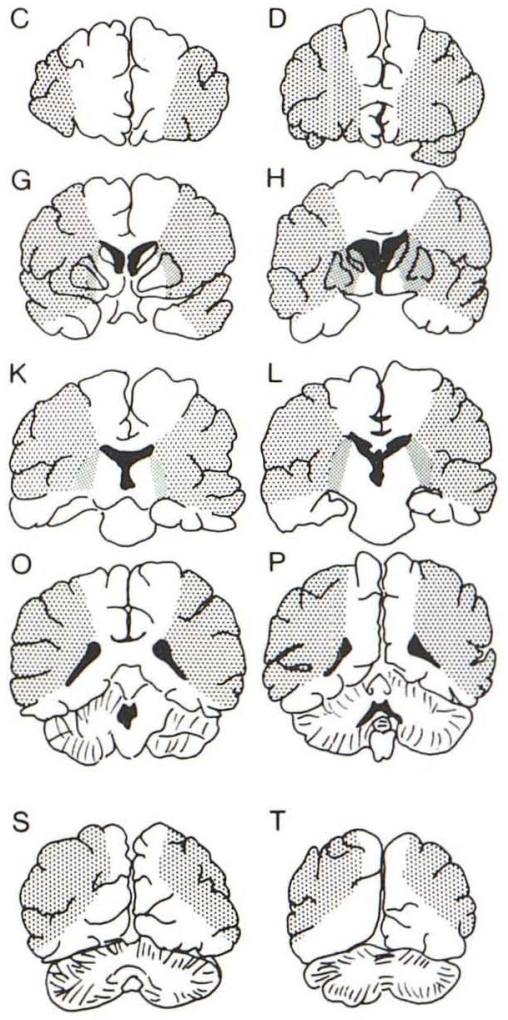

Fig. 2.-Coronal CT scan diagrams arranged in sequence from front to back. Angle and levels of scan planes are illustrated in figure 3 . Territories of middle cerebral artery are identified as in figure 1. 


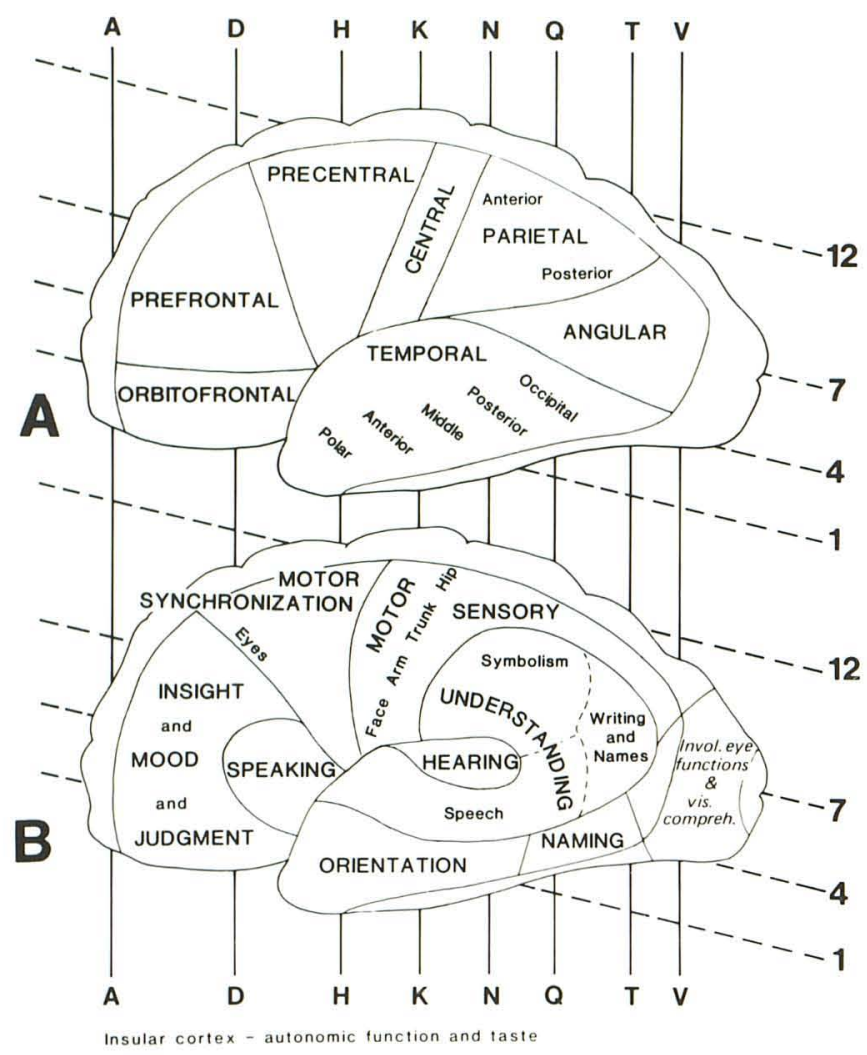

Fig. 3.-Arterial territories and functions correlated. A, Regions supplied by hemispheric branches of middle cerebral artery on lateral view of left cerebral hemisphere. Axial and coronal scan levels marked in figures 1 and 2 are indicated for cross-referencing. B, Functional cortical regions supplied by middle cerebral artery localized on lateral view of left cerebral hemisphere. Areas marked speaking and understanding are found only in dominant hemisphere (usually the left). These zones have poorly understood functions in nondominant hemisphere. Axial and coronal scan levels marked in figures 1 and 2 are indicated for correlation.

within the sylvian fissure. This infolded cortex is supplied by the major hemispheric branches that course over its surface. Small branches arise from these surface vessels and penetrate the insular cortex, extreme capsule, and claustrum.

Damage to the insular cortex can produce the opercular syndrome of Foix-Chavany-Marie, which is characterized by impairments of speech articulation (dysarthria) and clarity (dysphonia), swallowing (dysphagia), and chewing (dysmasesia) [18]. These problems are due to the interruption of signals to the fifth, seventh, and ninth through 12th cranial nerves. This syndrome is a type of pseudobulbar palsy. In contrast, direct damage to the nuclei of these nerves is termed bulbar palsy and is associated with flaccidity of the denervated muscles and loss of facial reflexes. Pseudobulbar palsies, on the other hand, produce increased tone and increased reflexes. Usually pseudobulbar palsies are caused by pontine or capsulostriatal lesions. These are often accompanied by mental impairment and loss of sphincter control. In the cortical (opercular) pseudobulbar palsy, the mental impairment and sphincter incontinence do not occur. Also, the increased tone is less marked or, often, absent. This syndrome is usually seen in cases with bilateral insular lesions [19].

The lateral surface of the frontal lobe mediates judgment, insight, and mood. It receives arterial supply from the orbito- frontal and prefrontal branches of the middle cerebral artery (the medial surface of the frontal lobe contains the remainder of the judgment, insight, and mood areas, which are supplied by the anterior cerebral artery) $[1,4,16,17]$.

The prefrontal and precentral arteries supply that area of the dominant hemisphere that is essential for "speaking" (fig. 3). Damage here causes Broca (motor) aphasia, which, as noted in the striate discussion, causes markedly reduced verbal output (agrammatic and telegraphic) and impaired ability to repeat sentences and phrases or name objects [14, 15]. The patient's ability to understand speech remains intact. An example of a lesion in this region is shown in figure 6 .

A much less common type of aphasia, transcortical motor aphasia, results from lesions rostral or superior to Broca area $[14,20]$. Infarctions of the territories of the orbitofrontal, prefrontal, and precentral middle cerebral arterial branches (fig. 3A) or a watershed infarction of the zone between the anterior and middle cerebral arteries may produce this syndrome. The patient suffers an aphasia identical to Broca except that repetition of sentences and phrases is not impaired $[15,18]$.

As shown in figure 3 , the precentral artery also supplies those parts of the frontal lobe that facilitate "motor synchronization." One area in this region controls coordination of eye and head movement. Damage here deprives the patient of the ability to look in the direction opposite the side of the lesion [21].

The cortex marked "motor" in figure $3 \mathrm{~A}$ is supplied by the central branch of the middle cerebral artery. Damage in this territory causes contralateral weakness of face, arm, trunk, and hip. The motor cortex of the medial surface of the frontal lobe that controls the leg is supplied by the anterior cerebral artery [1]. The leg, however, may be weakened after occlusion of the central branch of the middle cerebral artery. This occurs if the lesion also damages the motor fiber tracts to the leg as they travel downward beneath the cortical areas (marked "face" and "arm" in figure 3B). It may also occur when the cortical middle cerebral lesion causes edema in the medial front lobe, which compresses the motor fiber tracts of the leg. The leg weakness associated with middle cerebral cortical lesions is usually less severe and more transient than that seen with anterior cerebral artery infarcts of the cortex, which controls motor function of the leg $[1,6,16,17,21]$.

The cortex marked "sensory" in figure 3B is supplied by central and anterior parietal branches [7]. Damage to this region causes loss of high-order sensory functions in the face, arm, trunk, and hip. Occasionally the leg is involved, presumably by the mechanisms described previously. These lesions cause loss of: (1) position sense; (2) tactile localization; (3) stereognosis; (4) ascertainment of shape, size, and texture; (5) two point discrimination; and (6) recognition of letters or numbers written on the skin (graphesthesia) [6, 21, 22]. This cortical sensory loss contrasts with loss of primary sensory perception (pain, temperature, vibration, and touch) found with lesions in the lateral striate territory of the middle cerebral artery.

The posterior parietal, angular, occipital, and posterior temporal arteries shown in figure 3 vascularize a part of the cortex that, in the dominant hemisphere, mediates "understanding" $[4,17]$. Damage to this region, which is known as 


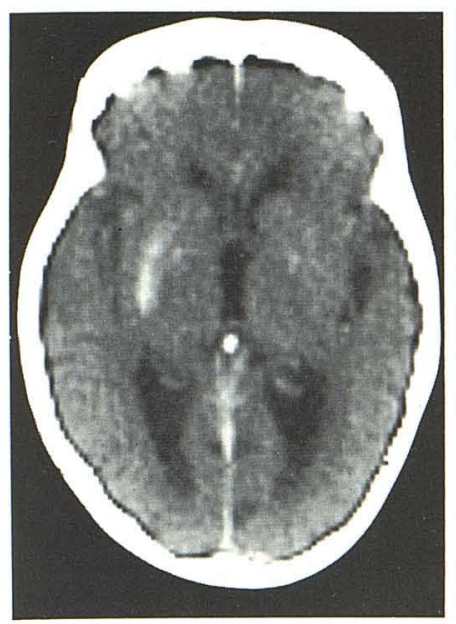

A

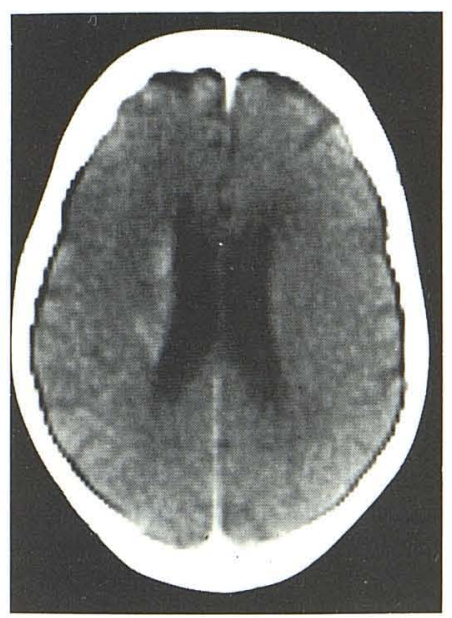

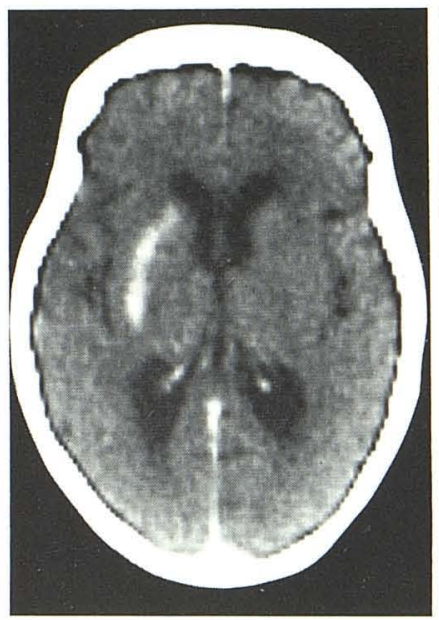

B

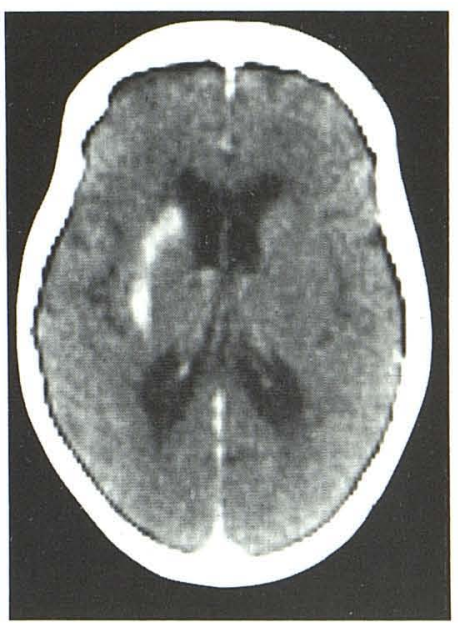

C

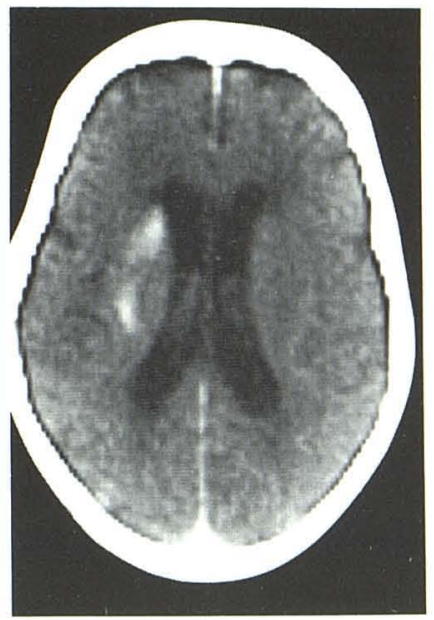

D

Fig. 4.- Infarction of lateral part of striate territory of middle cerebral artery. Axial CT scans corresponding to levels 4-7 of figure 1 demonstrate contrast enhancement. Sparing of anterior inferior caudate nucleus, which is supplied by medial lenticulostriate branch of anterior cerebral artery (i.e., recurrent artery of Heubner) and/or anterior choroidal artery. This lesion produced loss of sensory and motor function of left side of face as well as left arm. Left leg was affected because there was damage to motor fibers as they descended and entered posterior limb of internal capsule.

E

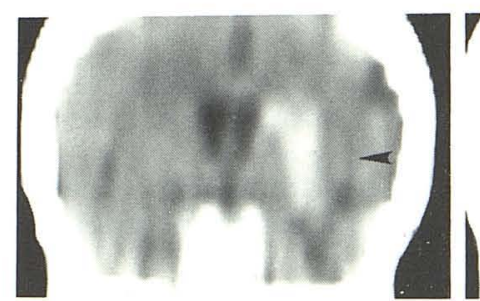

A

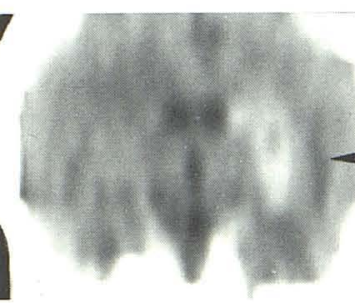

B

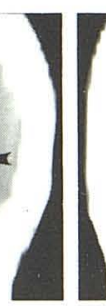

C

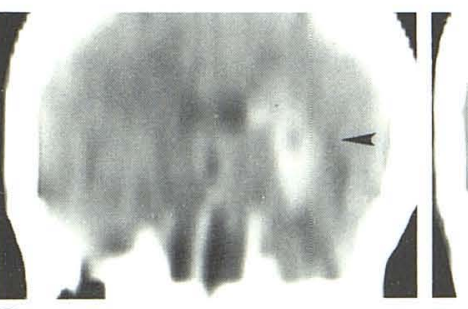

Fig. 5.- Infarction of outermost branches of lateral lenticulostriate territory. Coronal reconstruction corresponding to levels $\mathrm{H}-\mathrm{L}$ in figure 3 shows contrast enhancement. Brain medial to sylvian fissure (arrowheads) (i.e., insula, claustrum, and extreme capsule) is supplied by hemispheric branches and is not

Wernicke area, causes striking neuropsychologic symptoms $[6,17,21]$. Involvement of the lower part of the area impairs perception and understanding of speech. Damage to the rest of it impairs written, visual, and auditory language integration $[14,15]$. Lesions tend to involve the entire Wernicke area, producing an aphasia in which language comprehension, repetition, and naming are all severely affected, though the spontaneity and fluency of speech remain intact. As a result, the patient with Wernicke aphasia speaks meaningless gibberish that has normal intonation and fluency and he cannot understand written or spoken language $[14,15]$.

Lesions in part of the dominant hemisphere supplied by the

compromised by striate infarctions. Brain medial to infarct at level in $\mathbf{A}$ is supplied by medial striate branches of anterior cerebral artery (i.e., recurrent artery of Heubner) and anterior choroidal artery. Brain medial to infarct at levels in $\mathbf{B}-\mathbf{D}$ is supplied by uninvolved thalamic branches of posterior cerebral artery.

angular artery may produce a neuropsychologic deficit known as Gerstmann syndrome. In this syndrome the patient exhibits a variety of problems in understanding. Acalculia (loss of arithmetical ability), alexia (inability to read), finger agnosia (inability to identify one's fingers), and right-left confusion may be present [23]. Aphasia is also sometimes encountered.

Infarction of the parietal lobe occurs when there is occlusion of the central, anterior, and/or posterior parietal arteries. In the nondominant hemisphere, it produces disturbances in the ability to utilize or synthesize geometric and spatial information (amorphosynthesis) [24]. These disturbances can take many forms. Some of them are syndromes of unilateral ne- 


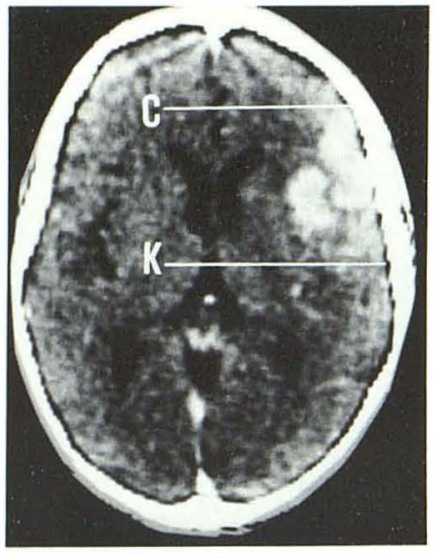

Fig. 6.- Infarction of territory of hemispheric branches of left middle cerebral artery. Axial CT scan corresponding to level 5 in figure 1 shows contrast enhancement extending from coronal plane just in front of genu of corpus callosum (level $\mathrm{C}$ in fig. 2) to midbody of third ventricle (level K). Area bracketed by these levels is in fig. 3A. Prefrontal, precentral, and central branches supply cortex at level 5 , in region defined by vertical lines $C-K$. This same area can be found in figure $3 \mathrm{~B}$ and used to predict patient's speech difficulty (Broca area), motor incoordination, and right facial numbness and paralysis. Cortex above scan level was preserved by collateral circulation from anterior cerebral artery.

glect. For example, the patient may ignore the existence of the half of the world contralateral to the lesion. Only part of such neglect may be due to concomitant lesions of the sensory cortex or involvement of the optic radiations, which produces a contralateral hemianopsia [25]. Other neglect syndromes are the denial of illness, hemiplegia, or pain in the contralateral body (anosagnosia). The patient may also have difficulty clothing himself, which is often worse contralateral to the lesion (dressing apraxia). These syndromes cannot be explained by concomitant paralysis, sensory loss, or dementia. Another difficulty that may be encountered in nondominant parietal lesions is loss of orientation or spatial memory. In these instances the subject has difficulty finding his way around the town, house, or room. All of the syndromes described for the nondominant parietal lobe may also be seen with lesions of the dominant parietal lobe, but they are difficult to detect because of concomitant aphasia [25].

The anterior temporal and temporal polar arteries supply the inferior temporal areas and a portion of the insular cortex. Infarction of these branches does not produce readily discernible clinical signs and symptoms, although autonomic disturbances such as contralateral mydriasis (enlargement of the pupil) and hyperhydrosis (increased perspiration) may be associated with damage in this area [21].

\section{Application}

The functional neuroanatomy and the vascular territories of the region supplied by the middle cerebral artery have been graphically summarized in a format directly applicable to CT to simplify correlation of CT images, clinical signs and symptoms, and vascular territories in everyday practice. Using this material the reader may, after finding a lesion at CT, choose the axial or coronal diagram or both that corresponds with the abnormal level(s) and determine whether the lesion is confined to a vascular territory. If it is, the schematic diagrams can be used to determine which branches supply the area. Broad functional correlation is then provided by an illustration that maps the function of the cortex. Further information outlining the fiber tracts encompassed within this territory can be gleaned from the accompanying text. We believe that using this information in the clinical situation can improve the usefulness and accuracy of CT in the evaluation of cerebral lesions.

\section{REFERENCES}

1. Berman SA, Hayman LA, Hinck VC. Correlation of cerebral vascular territories with cerebral function by computed tomography. I. Anterior cerebral artery. AJNR 1980;1:259-263, AJR 1980;135:253-257

2. Hayman LA, Berman SA, Hinck VC. Correlation of CT cerebral vascular territories with function. II. Posterior cerebral artery. AJNR 1981;2:219-225, AJR 1981;137:13-19

3. Matsui T, Hirano A. An atlas of the human brain for computerized tomography. Tokyo: Igaku-Shoin, 1978:314-547

4. Stephens RB, Stilwell DL. Arteries and veins of the human brain. Springfield, IL: Thomas, 1969:33-70

5. Gibo H, Carver CC, Rhonton AL, Lenkey C, Mitchell RJ. Microsurgical anatomy of the middle cerebral artery. I Neurosurg 1981;54:151-169

6. Vick N. Grinker's neurology, 7th ed. Springfield, IL: Thomas, 1976:482-486

7. Fisher CM. Capsular infarcts, the underlying vascular lesions. Arch Neurol 1979;36:65-73

8. Fisher $\mathrm{CM}$, Curry HB. Pure motor hemiplegia of vascular origin. Arch Neurol 1965;13:130-140

9. Mohr JP. Lacunes. Stroke 1982;13:3-11

10. Ho K. Pure motor hemiplegia due to infarction of the cerebral peduncle. Arch Neurol 1982;39:524-526

11. Fisher CM. Lacunar strokes and infarcts: a review. Neurology (NY) 1982;32:871-876

12. Rascol A, Clanet M, Manelfe C, Guiaud B, Bonofe A. Pure motor hemiplegia: CT study of 30 cases. Stroke 1982;13:11-17

13. Naeser MA, Hayward RW. Lesion localization in aphasia with cranial computed tomography and the Boston diagnostic aphasia exam. Neurology (NY) 1978;28:545-555

14. Benson DF, Geschwind N. The aphasias and related disturbances. In: Baker AB, Baker LH, eds. Hagerstown, MD: Harper \& Row, 1971: chapter 8

15. Goldstein K. Language and language disturbances. New York: Grune \& Stratton, 1948

16. Foix C, Levy M. Les ramollissement Sylviens, syndromes des lesions en foyer du territories de l'artere Sylviens et de ses branches. Rev Neurol (Paris) 1927;48:1-51

17. Waddington MM, Ring BA. Syndromes of occlusion of middle cerebral artery branches. Angiographic and clinical correlation. Brain 1968;91:685-696

18. Foix C, Chavany A, Marie P. Diplégie facio-linguo-masticatrice cortico-sous-corticale sans paralysie des membres. Rev Neurol (Paris) 1926;33:214-219

19. Bruyn GW, Gathier JC. The operculum syndrome. In: Vinken PJ Bruyn GW, eds. Handbook of clinical neurology, vol. 2. Amsterdam: North-Holland, 1969:776-783

20. Rubens AB. Transcortical motor aphasia. In: Whitaker $H$, Whitaker HA, eds. Studies in neurolinguistics, vol 1. New York: Academic Press, 1976:293-306

21. Adams RD, Victor M. Principles of neurology, $2 \mathrm{~d}$ ed. New York: McGraw-Hill, 1981:534-538

22. Dejerine J, Mouzon J. Un nouveau type de syndrome sensitif corticale observe dans un cas de monoplegie corticale dissociee. Rev Neurol (Paris) 1914;28:1265-1273

23. Gerstmann J. Zur Symptomatologie der Hirnlaswness im Ubergangpgebiet der unteren Parietal und mittleren Occipitalurilung. Nervenarzt 1930;3:691-695

24. Denny-Brown D, Banker B. Amorphosynthesis from left parietal lesions. Arch Neurol Psychiatry 1954;1:302-312

25. Critchley M. The parietal lobes. London: Arnold, 1953 